\title{
Linear relation between Heisenberg exchange and interfacial Dzyaloshinskii-Moriya interaction in metal films
}

\author{
Hans T. Nembach ${ }^{\star}$, Justin M. Shaw, Mathias Weiler ${ }^{\dagger}$, Emilie Jué and Thomas J. Silva
}

\begin{abstract}
Proposals for novel spin-orbitronic logic ${ }^{1}$ and memory devices ${ }^{2}$ are often predicated on assumptions as to how materials with large spin-orbit coupling interact with ferromagnets when in contact. Such interactions give rise to a host of novel phenomena, such as spin-orbit torques ${ }^{3,4}$, chiral spin structures $^{5,6}$ and chiral spin torques ${ }^{7,8}$. These chiral properties are related to the antisymmetric exchange, also referred to as the interfacial Dzyaloshinskii-Moriya interaction (DMI; refs 9,10 ). For numerous phenomena, the relative strengths of the symmetric Heisenberg exchange and the DMI are of great importance. Here, we use optical spin-wave spectroscopy (Brillouin light scattering) to directly determine the volumeaveraged DMI vector $D$ for a series of $\mathrm{Ni}_{80} \mathrm{Fe}_{20} / \mathrm{Pt}$ thin films, and then compare the nearest-neighbour DMI coupling energy with an independently measured value of the Heisenberg exchange for each sample. We show that the dependence on $\mathrm{Ni}_{80} \mathrm{Fe}_{20}$ thickness of both the microscopic symmetric and antisymmetric exchange are nearly identical, consistent with the notion that the fundamentals of the DMI and Heisenberg exchange essentially share the same underlying physics, albeit with different symmetries, as was originally proposed by Moriya ${ }^{11}$ for superexchange in magnetic oxides, and by Fert and Levy ${ }^{12}$ for RKKY coupling in metallic spin glasses. Indeed, our result demonstrates the generality of the original DMI theory, insofar as the proportionality of the symmetric and antisymmetric exchange is robust with regard to the details of spin coupling for the material system in question. Although of significant fundamental importance, this result also leads us to a deeper understanding of DMI and how it could be optimized for spin-orbitronic applications.
\end{abstract}

Recent experimental results have demonstrated how the interplay of symmetric (Heisenberg) exchange and antisymmetric (DMI) exchange, together with anisotropy, can give rise to a variety of magnetostatic phenomena, such as magnetic skyrmion lattices ${ }^{13}$, spiral spin structures ${ }^{14}$ and chiral domain walls ${ }^{6}$. In bilayer materials with a sufficiently thin, perpendicularly magnetized ferromagnet (FM) adjacent to a metal with large spin-orbit coupling in the conduction band, a large DMI favours Néel domain walls with a fixed chirality ${ }^{15}$, as opposed to Bloch walls. The combination of a chiral domain wall structure and spin-orbit torque can give rise to fast current-induced domain wall motion ${ }^{16}$. The direction and the speed are both dependent on the sign and the strength of the DMI and the spin-orbit torque ${ }^{7,8}$. Moreover, theory for a Rashba model predicts that the interfacial spin-orbit torque is proportional to the ratio of symmetric and antisymmetric exchange ${ }^{17}$. Thus, direct determination of both the DMI and Heisenberg exchange is crucial for an understanding of the underlying physics in such materials systems and a better understanding of the spin-orbit torques.

So far, direct measurements of antisymmetric exchange are limited to exotic measurement techniques that can only be applied to a few highly specialized sample systems. For example, the DMI constant has been measured by means of synchrotron-based X-ray scattering interferometry for the weak ferromagnet $\mathrm{FeBO}_{3}$ (ref. 18), by spin-polarized electron energy loss spectroscopy for an atomic bilayer of Fe on W(110) (ref. 19) and by spin-polarized scanning tunnelling microscopy for atomic monolayer Mn on W(110) (ref. 5). Until now, estimation of the antisymmetric exchange in the case of arbitrary materials has been possible only via inference from indirect measurement methods. These methods include both determination of the critical ferromagnetic layer thickness at the transition from a Néel to a Bloch wall ${ }^{6}$, and measurements of domain wall motion $^{20,21}$. Furthermore, quantitative experimental comparison of the symmetric and antisymmetric exchange is still outstanding.

Recent theory predicts an asymmetric dispersion shift of longwavelength thermal spin waves in magnetic thin films due to the DMI (refs 22-26). Motivated by this theory, we used Brillouin light scattering (BLS) to directly measure the predicted asymmetric dispersion shift, which in turn allowed us to determine the magnitude and direction of the DMI vector in a technological relevant sample system: we used a series of sputtered multilayer stacks consisting of $\mathrm{SiN} / \mathrm{Ni}_{80} \mathrm{Fe}_{20}(t) / \mathrm{Pt}(6 \mathrm{~nm}) / \mathrm{Ta}(3 \mathrm{~nm}) /$ substrate, where $t$ ranged from $1 \mathrm{~nm}$ to $13 \mathrm{~nm}$. We independently determined the symmetric exchange by fitting low-temperature magnetometry data to the Bloch $\mathrm{T}^{3 / 2}$ law for the same samples. Comparison of the two data sets allowed us to unambiguously determine the proportionality of the symmetric and antisymmetric exchange.

Interfacial DMI in a thin ferromagnetic film adjacent to a high spin-orbit material is mediated by an atom in the high spin-orbit material. The Hamiltonian for the DMI between two spins $\mathbf{S}_{i}$ and $\mathbf{S}_{j}$ on the atomic sites $i$ and $j$ is given by $H_{\mathrm{DMI}}=-\mathbf{D}_{i j} \cdot\left(\mathbf{S}_{i} \times \mathbf{S}_{j}\right)$. Here, $\mathbf{D}_{i j}=D_{i j} \mathbf{n} \times \mathbf{e}_{i j}$ is the DMI vector, which lies in the symmetrybreaking plane and is perpendicular to the unit vector that connects sites $i$ and $j, \mathbf{e}_{i j} \doteq \mathbf{r}_{i j} /\left|\mathbf{r}_{i j}\right|$, as shown in Fig. 1a,b, and $\mathbf{n}$ is the interface normal. Spin waves in the ferromagnet have a spatial chirality, which depends on their propagation direction with respect to the direction of the magnetization. In particular, for $\mathbf{M} \|+z$, spin waves propagating in the $-x$ direction have anticlockwise spatial chirality (Fig. 1a), whereas those propagating in the $+x$ direction have clockwise chirality (Fig. 1b). However, the spatial chirality favoured by the DMI is fixed by the sign of $D_{i j}$, thus the presence 


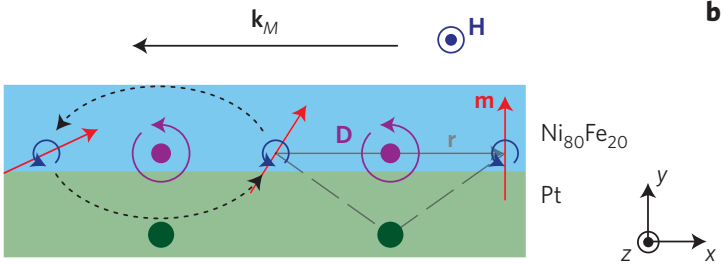

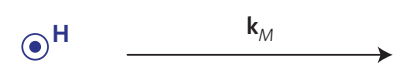

c
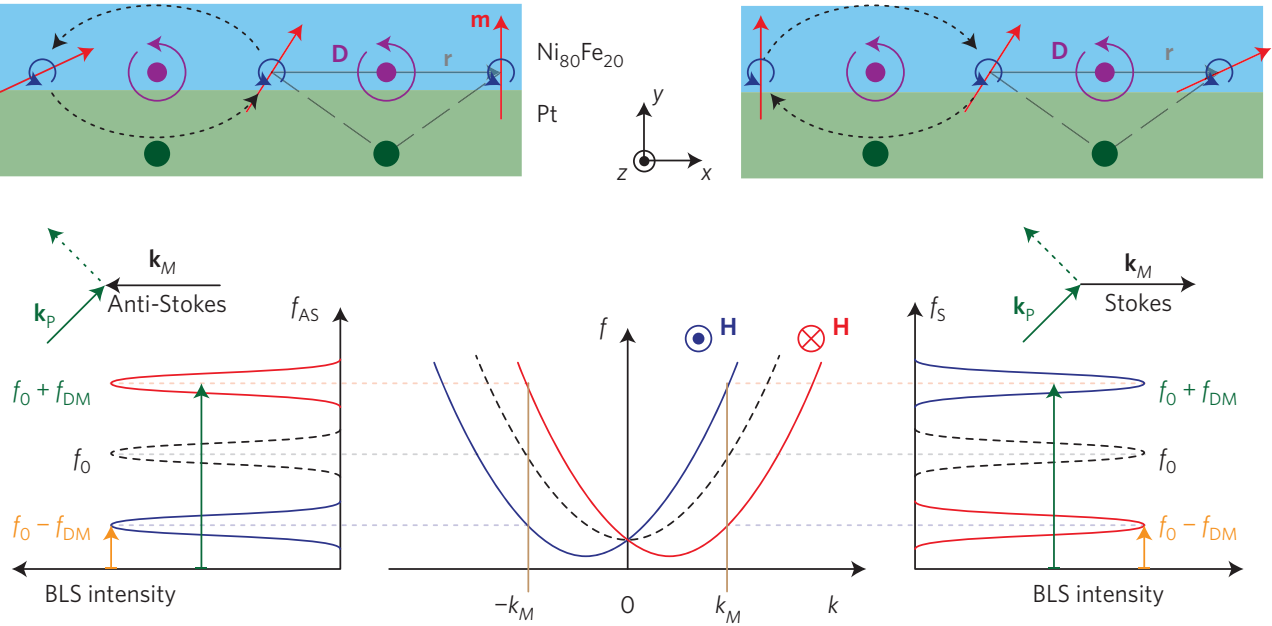

Figure 1 | Modification of spin-wave propagation in the presence of interfacial DMI. a, Sketch of a Damon-Eshbach spin wave propagating at the $\mathrm{Ni}_{80} \mathrm{Fe}_{20} / \mathrm{Pt}$ interface with wavevector $\mathbf{k}_{M} \|-x$ with the magnetization $\mathbf{M} \|+z$. All individual moments precess in the external field $\mathbf{H}$ in an identical (anticlockwise) manner, indicated by the blue arrows. The canted arrows depict the dynamic components of the spins at a snapshot in time. The dashed arrows indicate the spatial chirality of the spin wave along $x$. Any two neighbouring spins (red arrows), are coupled by a DMI vector D (purple vector pointing out of page) via a three-site exchange mechanism that includes a Pt atom (green atoms). The Pt atom serves to both break the symmetry and provide the necessary spin-orbit coupling. The preferred chirality of the antisymmetric exchange indicated by the purple arrow circulating about the DMI vector is identical to the spatial spin wave chirality. $\mathbf{b}$, For $\mathbf{k}_{M} \|+x$, the spatial chirality of the spin wave is opposite to that favoured by the DMI. The

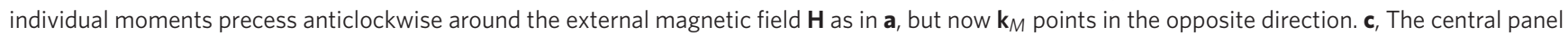

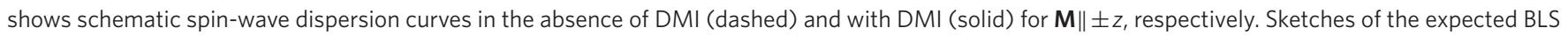
spectra for $-\mathbf{k}_{M}$ (anti-Stokes process, annihilation of a magnon, propagation in the $-x$-direction) and $+\mathbf{k}_{M}$ (Stokes process, generation of a magnon, propagation in the $+x$-direction) are shown on the left and right sides of the lower panel, respectively.

of the DMI causes an asymmetric modification of the spin-wave dispersion relation. For an in-plane magnetized film with spin waves propagating perpendicular to the magnetization direction, the DMI modifies the frequency

$$
f_{M}=f_{0}+\Delta f_{\mathrm{DMI}}
$$

of the spin waves ${ }^{22}$ (see middle panel of Fig. 1c.) Here, $f_{0}$ is the spinwave frequency in the absence of the DMI and

$$
\Delta f_{\mathrm{DMI}}=\left|\frac{g^{\|} \mu_{\mathrm{B}}}{h}\right| \operatorname{sgn}\left(M_{z}\right) \frac{2 D_{\mathrm{DMI}}}{M_{\mathrm{s}}} k_{x}
$$

is the DMI-induced frequency shift, where $D_{\mathrm{DMI}} \propto D_{i j}$ is the volumetric DMI constant that determines the sign and magnitude of the DMI vector, $g^{\|}$is the in-plane spectroscopic splitting factor, $M_{\mathrm{s}}$ the saturation magnetization, $\mathbf{k}$ (with magnitude $k$ ) the wavevector of the spin waves, $\mu_{\mathrm{B}}$ the Bohr magneton and $h$ Planck's constant. In the presence of the DMI, the spin-wave frequencies have a shift linear in $k_{x}$ to either higher or lower frequencies, depending of the propagation direction and the polarity of the static magnetization component $\mathbf{M}$. If the spin-wave spatial chirality is favoured by the DMI, the spin-wave frequency is reduced. Conversely, it is increased for the opposite chirality. Although the presence of surface anisotropy can also lead to a non-reciprocal spin-wave dispersion ${ }^{27}$, calculations presented in the Methods show that the asymmetric frequency shift of magnetostatic surface waves due to interfacial anisotropy is small for our measurements.

The dispersion characteristics of spin waves in thin magnetic films can be measured with BLS. In our BLS measurements, a laser is focused onto a sample, and the photons are inelastically backscattered by the quantized spin waves-that is, magnons. Momentum conservation dictates that magnons propagating towards the incoming laser beam must be annihilated (the anti-Stokes process), and magnons propagating in the opposite direction must be created (the Stokes process). (See Fig. 1c.) Energy conservation then uniquely identifies the inelastic energy shift of the backscattered photons with the magnon propagation direction: if the energy of the scattered photon is increased (or decreased) by the magnon energy, the direction of magnon propagation is either towards (or away from) the laser beam. Thus the measurement of the scattered photon energy can be used to determine the frequency difference of spin waves propagating in opposite directions, as sketched in Fig. 1c. We measured the spin-wave frequencies for the two field polarities transverse to the scattering plane, and for the two propagation directions. This yields four independent measurements of spin-wave frequency as a function of magnetization polarity and propagation direction. In Fig. 2a,b, normalized BLS spectra for $\mathrm{Ni}_{80} \mathrm{Fe}_{20}$ film samples of thickness $t=1.3 \mathrm{~nm}$ and $2.0 \mathrm{~nm}$, respectively, on a $6 \mathrm{~nm}$ Pt underlayer are shown. For comparison, we also show data for a control sample film of $2.0 \mathrm{~nm} \mathrm{Ni} \mathrm{Ni}_{80} \mathrm{Fe}_{20}$ without a Pt underlayer in Fig. 2c. The BLS spectra of the samples with a Pt underlayer exhibit a frequency shift of the order of $100 \mathrm{MHz}$ that changes sign with magnetization polarity or reversal of the spin-wave propagation direction. This frequency difference is larger for the sample with thinner $\mathrm{Ni}_{80} \mathrm{Fe}_{20}$, as expected for an interfacial source of symmetry breaking, whereas there is no frequency difference for the control sample, in agreement with the absence of interfacial DMI in this case.

In Fig. 3, we show the $t$-dependence of the frequency shift $\Delta f_{\text {DMI }}$ averaged over the four possible combinations of the magnetization polarity and propagation direction. Consistent with the interfacial nature of the DMI, $\Delta f_{\mathrm{DMI}}$ decreases with increasing $t$. According to equation (1), $M_{\mathrm{s}}$ and $g^{\|}$are required to determine $D_{\text {DMI }}$ from the measured $\Delta f_{\text {DMI }} . M_{s}$ at $300 \mathrm{~K}$, measured by means of superconducting quantum interference device (SQUID) magnetometry, is shown in Fig. 4a. $g^{\|}$is measured by ferromagnetic 

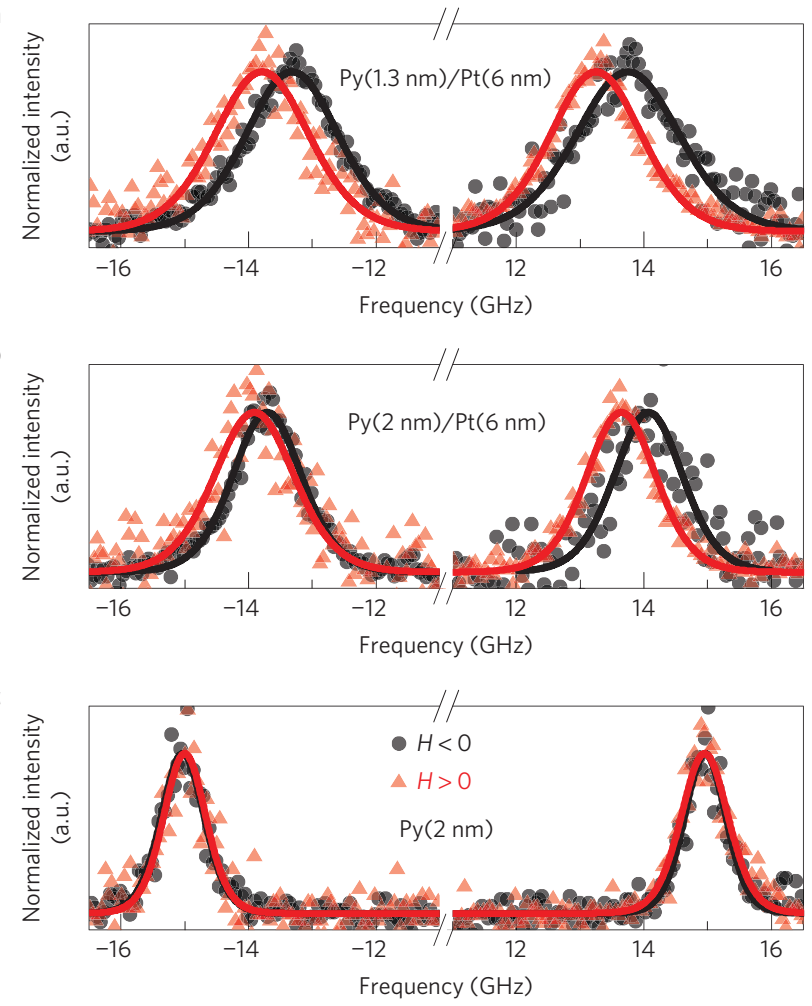

Figure 2 | Normalized spin-wave spectra, as measured by BLS. The measurements were carried out for the two opposite magnetization polarities (circles and triangles). The lines are fits to the data using the transmission function of the tandem multi-pass interferometer in the BLS spectrometer: $\left(\left(f-f_{0}\right)^{2}+\Delta f^{2}\right)^{-6}$. The peak positions correspond to the frequencies of Stokes (negative frequencies) and anti-Stokes (positive frequencies) processes with a fixed wavevector of $|\mathbf{k}|=16.7 \mu \mathrm{m}^{-1}$. a, Data for a $1.3 \mathrm{~nm} \mathrm{Ni} 80 \mathrm{Fe}_{20}$ film with a $6 \mathrm{~nm}$ Pt underlayer with $\mu_{0} \mathrm{H}= \pm 295 \mathrm{mT}$. b, Data for a $2.0 \mathrm{~nm} \mathrm{Ni} 80 \mathrm{Fe}_{20}$ film with a $6 \mathrm{~nm}$ Pt underlayer with $\mu_{0} H= \pm 224 \mathrm{mT}$. The spin-wave frequency is clearly shifted by reversal of the magnetization direction in both $\mathbf{a}$ and $\mathbf{b}$, and the frequency shift is reduced for the sample with $2.0 \mathrm{~nm}$ of $\mathrm{Ni}_{80} \mathrm{Fe}_{20}$. c, Data for a reference sample without Pt with $\mu_{0} H= \pm 295 \mathrm{mT}$ do not show a frequency shift that depends on either the magnetization direction or the spin-wave propagation direction.

resonance spectroscopy (see Supplementary Information). We use equation (1) to calculate $D_{\mathrm{DMI}}$, shown in Fig. $4 \mathrm{~b}$. Under the assumption that the DMI is a strictly interfacial property that is not affected by the bulk properties of the magnetic film, the magnitude of $D_{\text {DMI }}$ should be linearly proportional to $1 / t$ and be zero in the limit of an infinitely thick film. However, the measured $D_{\mathrm{DMI}}$ exhibits a nonlinear dependence on $1 / t$. This result implies that the DMI strength at the interface itself changes with the film thickness. Under the assumption that only the first monolayer of $\mathrm{Ni}_{80} \mathrm{Fe}_{20}$ at the $\mathrm{Pt}$ interface contributes to the antisymmetric exchange, the strength of the DMI at the interface $D_{\mathrm{DMI}}^{\mathrm{int}}$ can be calculated from

$$
D_{\mathrm{DMI}}^{\mathrm{int}}=D_{\mathrm{DMI}} \frac{\sqrt{3}}{a} t
$$

where $a=0.354 \mathrm{~nm}$ is the lattice constant for $\mathrm{Ni}_{80} \mathrm{Fe}_{20}$. $D_{\mathrm{DMI}}^{\mathrm{int}}$ is shown in Fig. 4c (left scale) and has a non-trivial dependence on the $\mathrm{Ni}_{80} \mathrm{Fe}_{20}$ thickness. In particular, we find that the magnitude of the antisymmetric exchange varies by a factor of almost 2.5 over the range of sample thicknesses. Using equation (6) in the Methods, the largest value for the interatomic antisymmetric exchange energy $D_{\mathrm{nn}}$

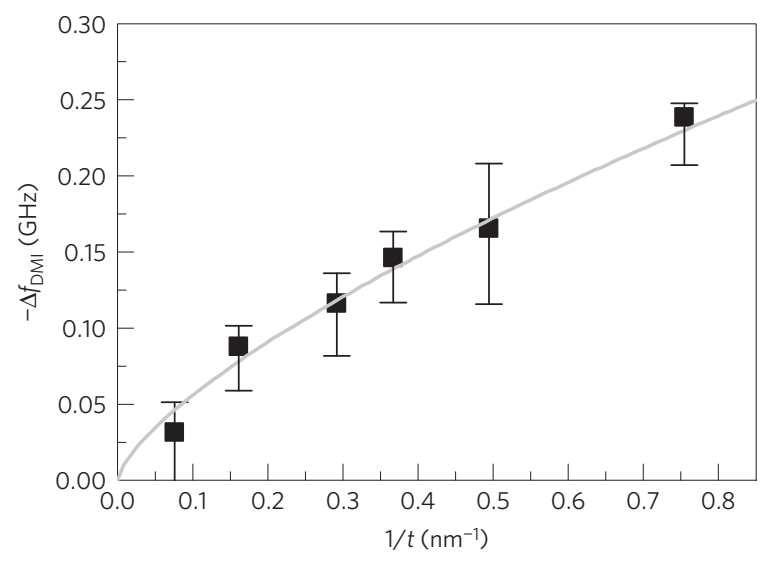

Figure 3 | DMI-induced spin-wave frequency shift. Frequency shift $\Delta f_{\mathrm{DMI}}$ as a function of the reciprocal $\mathrm{Ni}_{80} \mathrm{Fe}_{20}$ thickness. The grey line is a guide to the eye. $\Delta f_{D M I}$ increases with $1 / t$, in agreement with expectations derived from the interfacial nature of the DMI. The wavevector is given by the measurement geometry and is fixed at $|\mathbf{k}|=16.7 \mu \mathrm{m}^{-1}$. The error bars include an upper bound for the frequency shift due to non-reciprocal spin-wave dispersion of magnetostatic surface waves in the presence of surface anisotropy. Any out-of-plane surface anisotropy will reduce the DMI-induced frequency shift, and therefore results in asymmetric error bars.

that we obtain (for $t=6.1 \mathrm{~nm}$ ) is $-2.2_{-0.4}^{+0.7} \mathrm{meV}$. This is very similar in magnitude to the recently reported result of $+0.9 \mathrm{meV}$ for Fe/W in ref. 19 and $-1.05 \mathrm{meV}$ for a $\mathrm{Co} / \mathrm{Ni}$ multilayer on $\mathrm{Pt}(111)$ in ref. 6.

Theoretical calculations for the Dzyaloshinskii-Moriya interaction in bulk magnetic oxides and metallic spin glasses with magnetic impurities predict that the symmetric and antisymmetric exchange are proportional to each other ${ }^{11,12,28}$. It is therefore possible to speculate that the thickness dependence of $D_{\mathrm{DMI}}^{\mathrm{int}}$ is the result of a coincidental thickness dependence of the symmetric exchange for this particular system. The Heisenberg exchange Hamiltonian that describes the interaction of two spins is given by $H_{\text {ex }}=-J_{i j} \mathbf{S}_{i} \cdot \mathbf{S}_{j}$, with the exchange integral $J_{i j}=2 g^{\|} \mu_{\mathrm{B}} D_{\text {spin }} /\left(M_{\mathrm{s}} a^{5}\right)$ for the fcc lattice, where $D_{\text {spin }}$ is the spin-wave stiffness. Given that $J_{i j}$ is strongly influenced by the local environment of the electron wavefunction that overlaps sites $i$ and $j$, it is always plausible that there is a non-trivial thickness dependence for the volume-averaged exchange. As we show below, this is indeed the case for our particular sample system.

We measure the temperature dependence of the saturation magnetic moment $m_{\mathrm{s}}(T)$ with a SQUID magnetometer and fit the data with the Bloch $T^{3 / 2}$ law at low temperatures ${ }^{29}$,

$$
m_{s}(0 \mathrm{~K})-m_{s}(T) \propto\left(\frac{k_{\mathrm{B}} T}{D_{\text {spin }}^{0 \mathrm{~K}}}\right)^{3 / 2}
$$

where $T$ is the temperature, $D_{\text {spin }}^{0 \mathrm{~K}}$ is the low-temperature spin-wave stiffness, and $k_{\mathrm{B}}$ is the Boltzmann constant ${ }^{29}$. To determine the exchange constant $A$ at $300 \mathrm{~K}$, we use

$$
A \doteq A(T=300 \mathrm{~K})=\frac{M_{s}^{300 \mathrm{~K}} D_{\text {spin }}^{300 \mathrm{~K}}}{2 g^{\|} \mu_{\mathrm{B}}}
$$

where we take the temperature-dependent renormalization of the spin-wave stiffness into account, as detailed in the Supplementary Information. In Fig. 4c (right scale), the thickness dependence of $A$ is shown. Although the microscopic origins of the variation in the symmetric exchange with film thickness is unclear, it is an empirical fact that both the symmetric exchange $A$ and the antisymmetric 
a

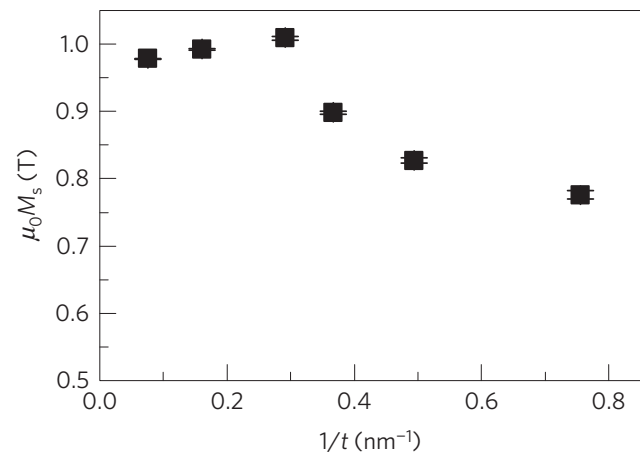

b

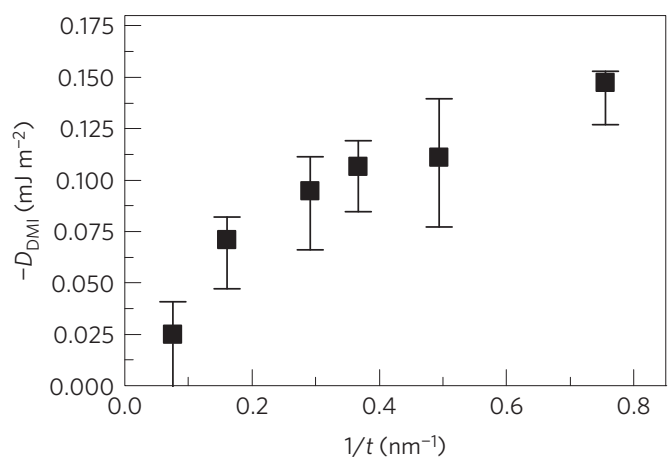

C

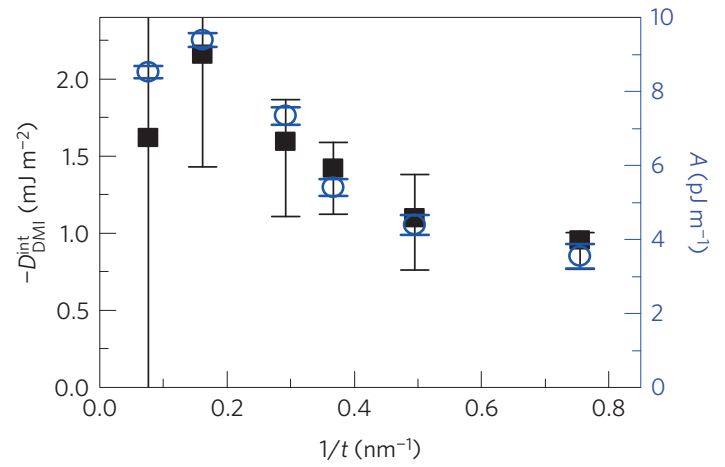

Figure 4 | Thickness dependence of the symmetric and the antisymmetric exchange. a, Thickness dependence of the magnetization, $M_{\mathbf{s}}$, at $300 \mathrm{~K}$. (The error bars are smaller than the symbol size.) b. The volumetric DMI $D_{\text {DMI }}$ does not follow a strict $1 / t$ dependence. c, The antisymmetric exchange $D_{\mathrm{DMl}}^{\text {int }}$ at the $\mathrm{Ni}_{80} \mathrm{Fe}_{20} / \mathrm{Pt}$ interface (squares, left scale) and the symmetric exchange $A$ in the $\mathrm{Ni}_{80} \mathrm{Fe}_{20}$ bulk (open circles, right scale) are linearly proportional over the entire range of measured thicknesses. The proportionality of $A$ and $D_{\mathrm{DMl}}^{\mathrm{int}}$ was originally predicted by Moriya for magnetic oxides ${ }^{11}$ and by Fert and Levy for metallic spin glasses ${ }^{12}$. (The error bars for $D_{\mathrm{DMI}}$ and $D_{\mathrm{DMI}}^{\mathrm{int}}$ are the propagated errors of the averaged frequency shift. The error bars for $A$ reflect the variation of $A$ when using different exponents for the renormalization of exchange at non-zero temperatures, as explained in the Supplementary Information.)

exchange $D_{\mathrm{DMI}}^{\text {int }}$ exhibit the same non-trivial functional dependence on reciprocal thickness. If we consider only nearest-neighbour interactions, the ratio $D_{\mathrm{DMI}} / A$ is proportional to the ratio of the nearest-neighbour exchange integral $J_{\mathrm{nn}}$ and the magnitude of the nearest-neighbour DMI $D_{\mathrm{nn}}$,

$$
\frac{D_{\mathrm{nn}}}{J_{\mathrm{nn}}}=\frac{2 \sqrt{2} a}{3} \cdot \frac{D_{\mathrm{DMI}}^{\mathrm{int}}}{A}
$$

as shown in the Methods. From $D_{\mathrm{DMI}}^{\mathrm{int}}$ and $A$ in Fig. $4 \mathrm{c}$, we find by fitting equation (3) to the data that $D_{\mathrm{nn}} / J_{\mathrm{nn}}=-(8.4 \pm 0.3) \%$, independent of $\mathrm{Ni}_{80} \mathrm{Fe}_{20}$ thickness. Any potential existence of a gradient in the magnitude of the symmetric exchange $A$ through the thickness of the $\mathrm{Ni}_{80} \mathrm{Fe}_{20}$ can only be very small and would only marginally affect the proportionality between $D_{\mathrm{nn}}$ and $J_{\mathrm{nn}}$. The homogeneity of the exchange is exemplified by the fact that the volume-averaged magnetization for the $3.4 \mathrm{~nm}$ (16.6 monolayers) sample is already equal to the bulk value of $\mu_{0} M_{\mathrm{s}} \sim 1 \mathrm{~T}$ at $300 \mathrm{~K}$. The linear proportionality between the symmetric and antisymmetric exchange was first proposed for bulk materials in the original theory of Moriya ${ }^{11}$. Similarly, such a proportionality was also predicted by Fert et al. ${ }^{12,28}$, for metallic spin-glass systems. Our results confirm that this proportionality also applies to two-dimensional systems at the interface between a ferromagnetic layer and a material with large spin-orbit coupling.

An important implication of our result is the independence of interfacial chiral ordering on parameters that affect the Heisenberg exchange, as the spin canting angle at adjacent atomic sites is proportional to the ratio $D_{\mathrm{DMI}}^{\mathrm{int}} / A$. Thus, the methods and results presented here not only serve to elucidate the fundamental properties of the DMI, but may also have a substantial impact on the methodology to successfully develop spin-orbitronic devices.

Note added in proof: During the review process further evidence of the non-reciprocal spin-wave dispersion due to DMI has emerged: Phys. Rev. Lett., 114, 047201 (2015); Phys. Rev. B, 91, 214409 (2015); Phys. Rev. B, 91, 180405(R) (2015); Appl. Phys. Lett., 106, 052403 (2015).

\section{Methods}

Methods and any associated references are available in the online version of the paper.

Received 23 October 2014; accepted 29 June 2015; published online 3 August 2015

\section{References}

1. Bhowmik, D., You, L. \& Salahuddin, S. Spin Hall effect clocking of nanomagnetic logic without a magnetic field. Nature Nanotech. 9, 59-63 (2013).

2. Parkin, S. S. P., Hayashi, M. \& Thomas, L. Magnetic domain-wall racetrack memory. Science 320, 190-194 (2008).

3. Miron, I. M. et al. Perpendicular switching of a single ferromagnetic layer induced by in-plane current injection. Nature 476, 189-193 (2011).

4. Liu, L. et al. Spin-torque switching with the giant spin Hall effect of tantalum. Science 336, 555-558 (2012).

5. Bode, M. et al. Chiral magnetic order at surfaces driven by inversion asymmetry. Nature 447, 190-193 (2007).

6. Chen, G. et al. Tailoring the chirality of magnetic domain walls by interface engineering. Nature Commun. 4, 2671 (2013).

7. Ryu, K.-S., Thomas, L., Yang, S.-H. \& Parkin, S. Chiral spin torque at magnetic domain walls. Nature Nanotech. 8, 527-533 (2013)

8. Emori, S., Bauer, U., Ahn, S.-M., Martinez, E. \& Beach, G. S. D. Current-driven dynamics of chiral ferromagnetic domain walls. Nature Mater. 12, 611-616 (2013).

9. Dzyaloshinsky, I. A thermodynamic theory of 'weak' ferromagnetism of antiferromagnetics. J. Phys. Chem. Solids 4, 241-255 (1958).

10. Moriya, T. New mechanism of anisotropic superexchange interaction. Phys. Rev. Lett. 4, 228-230 (1960).

11. Moriya, T. Anisotropic superexchange interaction and weak ferromagnetism. Phys. Rev. 120, 91-98 (1960).

12. Fert, A. \& Levy, P. M. Role of anisotropic exchange interactions in determining the properties of spin-glasses. Phys. Rev. Lett. 44, 1538-1541 (1980).

13. Mühlbauer, S. et al. Skyrmion lattice in a chiral magnet. Science $\mathbf{3 2 3}$, 915-919 (2009).

14. Ferriani, P. et al. Atomic-scale spin spiral with a unique rotational sense: $\mathrm{Mn}$ monolayer on W(001). Phys. Rev. Lett. 101, 027201 (2008).

15. Thiaville, A., Rohart, S., Jué, É., Cros, V. \& Fert, A. Dynamics of Dzyaloshinskii domain walls in ultrathin magnetic films. Europhys. Lett. 100, 57002 (2012).

16. Miron, I. M. et al. Fast current-induced domain-wall motion controlled by the Rashba effect. Nature Mater. 10, 419-423 (2011).

17. Kim, K.-W., Lee, H.-W., Lee, K.-J. \& Stiles, M. D. Chirality from interfacial spin-orbit coupling effects in magnetic bilayers. Phys. Rev. Lett. 111, 216601 (2013). 
18. Dmitrienko, V. E. et al. Measuring the Dzyaloshinskii-Moriya interaction in a weak ferromagnet. Nature Phys. 10, 202-206 (2014).

19. Zakeri, K. et al. Asymmetric spin-wave dispersion on Fe(110): Direct evidence of the Dzyaloshinskii-Moriya interaction. Phys. Rev. Lett. 104, 137203 (2010).

20. Franken, J. H., Herps, M., Swagten, H. J. M. \& Koopmans, B. Tunable chiral spin texture in magnetic domain-walls. Sci. Rep. 4, 5248 (2014)

21. Je, S.-G. et al. Asymmetric magnetic domain-wall motion by the Dzyaloshinskii-Moriya interaction. Phys. Rev. B 88, 214401 (2013).

22. Moon, J.-H. et al. Spin-wave propagation in the presence of interfacial Dzyaloshinskii-Moriya interaction. Phys. Rev. B 88, 184404 (2013)

23. Kostylev, M. Interface boundary conditions for dynamic magnetization and spin wave dynamics in a ferromagnetic layer with the interface Dzyaloshinskii-Moriya interaction. J. Appl. Phys. 115, 233902 (2014).

24. Costa, A. T., Muniz, R. B., Lounis, S., Klautau, A. B. \& Mills, D. L. Spin-orbit coupling and spin waves in ultrathin ferromagnets: The spin-wave Rashba effect. Phys. Rev. B 82, 014428 (2010).

25. Udvardi, L. \& Szunyogh, L. Chiral asymmetry of the spin-wave spectra in ultrathin magnetic films. Phys. Rev. Lett. 102, 207204 (2009).

26. Cortés-Ortuño, D. \& Landeros, P. Influence of the Dzyaloshinskii-Moriya interaction on the spin-wave spectra of thin films. J. Phys. Condens. Matter 25, 156001 (2013).

27. Hillebrands, B. Spin-wave calculations for multilayered structures. Phys. Rev. B 41, 530-540 (1990)
28. Fert, A. Magnetic and transport properties of metallic multilayers. Mater. Sci. Forum 59-60, 439-480 (1990)

29. Vaz, C. a. F., Bland, J. a. C. \& Lauhoff, G. Magnetism in ultrathin film structures. Rep. Prog. Phys. 71, 056501 (2008)

\section{Acknowledgements}

The authors are grateful to Ward Johnson for his support of our BLS measurements, and thank M. Stiles and R. McMichael for stimulating discussions. M.W. acknowledges a stipend by the German academic exchange service (DAAD).

\section{Author contributions}

H.T.N. conceived the experiment, performed the BLS measurements and analysed the BLS data. J.M.S. fabricated and characterized the samples and performed SQUID measurements, M.W. performed the FMR measurements and analysis. All authors contributed to the interpretation of the results and writing of the manuscript.

\section{Additional information}

Supplementary information is available in the online version of the paper. Reprints and permissions information is available online at www.nature.com/reprints.

Correspondence and requests for materials should be addressed to H.T.N.

\section{Competing financial interests}

The authors declare no competing financial interests. 


\section{Methods}

Sample preparation. All samples were prepared by d.c. magnetron sputtering in an Ar base pressure of $\sim 0.07 \mathrm{~Pa}(\sim 0.5 \mathrm{mtorr})$ and a chamber base pressure of $3 \times 10^{-6} \mathrm{~Pa}\left(2 \times 10^{-8}\right.$ torr $)$. Samples were rotated at $1-2 \mathrm{~Hz}$ during deposition to eliminate growth-induced anisotropy. The deposition rates were calibrated by $\mathrm{X}$-ray reflectometry. The $\mathrm{SiN}(5 \mathrm{~nm}-10 \mathrm{~nm}) / \mathrm{Ni}_{80} \mathrm{Fe}_{20}(t) / \mathrm{Pt}(6 \mathrm{~nm}) / \mathrm{Ta}(3 \mathrm{~nm}) /$ substrate films were deposited on thermally oxidized Si. The silicon nitride capping layer was sputter-deposited from a pure $\mathrm{Si}$ target in an $\mathrm{Ar}$ and $\mathrm{N}_{2}$ gas mixture. The Ta seed layer induced a strong (111)-texture of both the Pt and $\mathrm{Ni}_{80} \mathrm{Fe}_{20}$.

SQUID magnetometry. We measured in-plane hysteresis curves at room temperature to determine the saturation magnetization $M_{s}(300 \mathrm{~K})$ of our samples. We find that $M_{\mathrm{s}}(300 \mathrm{~K})$ decreases with decreasing $\mathrm{Ni}_{80} \mathrm{Fe}_{20}$ layer thickness. In addition, we measured the dependence of the magnetic moment $m_{\mathrm{s}}(T)$ on temperature $T$ while exposed to an applied field of $\mu_{0} H=10 \mathrm{mT}$. The saturation magnetization $M_{s}(0 \mathrm{~K})$ was determined by extrapolation of the fit of $m_{s}(T)$ to the Bloch $T^{3 / 2} \mathrm{law}^{29}$. To determine from these fits the spin-wave stiffness, $D_{\text {spin }}^{0 \mathrm{~K}}$, at $0 \mathrm{~K}$ for the $\mathrm{Ni}_{80} \mathrm{Fe}_{20}$ thickness series, we first calculate the density of excited magnons at a given temperature. This calculation takes into account finite-size effects due to the reduced thickness of the $\mathrm{Ni}_{80} \mathrm{Fe}_{20}$ layer. The room-temperature exchange constant $A$ is then determined using a mean-field approach for the temperature dependence of the exchange. The details on the determination of the exchange constant, renormalization and calculation of the magnon density are given in the Supplementary Information.

Brillouin light scattering spectroscopy. We use a Brillouin light scattering spectrometer with a six-pass, tandem Fabry-Perot interferometer to measure the thermal spin waves frequency at a fixed angle of incidence $\theta=45^{\circ}$. For our measurements the incident laser power was $40 \mathrm{~mW}$. A $\lambda=532 \mathrm{~nm}$ laser beam was focused on the sample with an $f / 1.2$ lens. Thus the wavevector of the measured spin waves is $k=16.7 \mu \mathrm{m}^{-1}$. The collimated backscattered light was spatially filtered with an aperture (14 $\mathrm{mm}$ in diameter) to reduce the wavenumber uncertainty.

Ferromagnetic resonance measurements. We employed ferromagnetic resonance spectroscopy with the external magnetic field both parallel and perpendicular to the sample plane to determine the respective spectroscopic splitting factors $g^{\perp}$ and $g^{\|}$and the perpendicular anisotropy field $H_{k}$. Details about the measurements are given in the Supplementary Information.

If not otherwise stated, all error bars and all uncertainties stated in the text are single standard deviation uncertainties.

Determination of the Dzyaloshinskii-Moriya exchange. The DMI Hamiltonian for two atoms at sites $i$ and $j$ with the spins $\boldsymbol{S}_{i}$ and $\boldsymbol{S}_{j}$, respectively, is given by

$$
H_{i j}=-\mathbf{D}_{i j} \cdot\left(\mathbf{S}_{i} \times \mathbf{S}_{j}\right)
$$

where $\mathbf{D}_{i j} \propto \mathbf{r}_{i j} \times \hat{n}$ is the Dzyaloshinskii-Moriya vector, with $\mathbf{r}_{i j}$ being the vector between sites $i$ and $j$ and $\hat{n}$ is a unit vector along the axis of broken symmetry-that is, the interface normal-for our particular case. The following calculation applies to a single ferromagnetic monolayer in contact with a high spin-orbit material. Following the analysis of Udvardi and Szunyogh, for the case where a spin wave propagates perpendicular to the magnetization, the DMI contribution to the spin-wave energy for an atomic monolayer is ${ }^{25}$

$$
E_{\mathrm{DMI}}^{\mathrm{int}}=-c S \sum_{i \neq j}\left(\mathbf{D}_{i j} \cdot \hat{m}\right) \sin \left(\mathbf{k} \cdot \mathbf{r}_{i j}\right)
$$

where $\hat{m} \doteq \mathbf{M} / M_{\mathrm{s}}, \mathbf{k}$ is the spin-wave wavevector, $S$ is the total spin on each site, an $c= \pm 1$ is the spin-wave chirality. (The necessary factor $S$ is missing in the original version of equation (4) in ref. 25.) The chirality is defined by the orientation of the magnetic moment at each lattice site $e^{25}$ :

$$
\mathbf{e}_{i}(\mathbf{k}, c)=\mathbf{n}_{1} \cos \left(\mathbf{k} \cdot \mathbf{R}_{i}\right) \sin (\theta)+c \mathbf{n}_{2} \sin \left(\mathbf{k} \cdot \mathbf{R}_{i}\right) \sin (\theta)+\hat{m} \cos (\theta)
$$

Here, $\mathbf{R}_{i}$ is the position vector for the atomic site $i, \theta$ is the relative angle of the moments and $\hat{m}, \mathbf{n}_{1} \perp \hat{m}$ and $\mathbf{n}_{2}=\mathbf{n}_{1} \times \hat{m}$ are unit vectors. In a (111)-textured fcc crystal, the grains are randomly rotated around the surface normal and equation (4) needs to be averaged about all possible orientations. Evaluation of equation (4) in the long-wavelength limit then yields

$$
\begin{aligned}
E_{\mathrm{DMI}}^{\mathrm{int}} & \cong \frac{3 c D_{\mathrm{nn}} S a k}{\pi \sqrt{2}} \int_{0}^{2 \pi} \cos ^{2}(\phi) \mathrm{d} \phi \\
& =\frac{3}{\sqrt{2}} c D_{\mathrm{nn}} S a k
\end{aligned}
$$

where $\phi$ is the angle between $\mathbf{r}_{i j}$ and $\mathbf{k}, D_{\mathrm{nn}}$ is the magnitude of the nearest-neighbour DMI vector, and $a$ is the lattice constant.

Damon-Eshbach modes with antisymmetric exchange. Following the analysis of Moon et al. ${ }^{22}$, the spin-wave dispersion for spin waves in a thin film with $\mathbf{k} \perp \mathbf{M}$ for in-plane magnetization - that is, the Damon-Eshbach mode (DE) - in the presence of interfacial DMI is ${ }^{22-26,30,31}$

$$
\begin{aligned}
\omega= & \frac{\mu_{0} \mu_{\mathrm{B}} g^{\|}}{\hbar} \sqrt{\left(H+\frac{2 A}{\mu_{0} M_{\mathrm{s}}} k^{2}-H_{k}+M_{\mathrm{s}} \frac{1-\mathrm{e}^{-t|k|}}{t|k|}\right)} \\
& \times \sqrt{\left(H+\frac{2 A}{\mu_{0} M_{\mathrm{s}}} k^{2}+M_{\mathrm{s}}\left(1-\frac{1-\mathrm{e}^{-t|k|}}{t|k|}\right)\right)} \\
& +\operatorname{sgn}(M) \frac{\mu_{\mathrm{B}} g^{\|}}{\hbar} \cdot \frac{2 D_{\text {DMI }}}{M_{\mathrm{s}}} k
\end{aligned}
$$

where $A$ is the exchange constant. The last term in equation (5) accounts for the DMI, which causes a frequency shift linear in the spin-wave wavenumber $k$. The sign of the DMI-induced frequency shift depends on the sign of $D_{\mathrm{DMI}}$, the orientation of the magnetization (sign of $M$ ) and the propagation direction of the spin waves (sign of $k$ ). Here we are using a volumetric DMI constant instead of including the DMI into the boundary conditions. The results for these two approaches are in good agreement for $|\mathbf{k}| t<0.28$, which is the case for our measurements $\mathrm{s}^{23}$. Comparison with equation (5), and accounting for the conversion from the interfacial DMI to the volumetric DMI, as quantified in equation (2), allows us to express the volumetric DMI constant $D_{\mathrm{DMI}}$ in terms of the microscopic $D_{\mathrm{nn}}$ as

$$
D_{\mathrm{DMI}}=\frac{3}{2 \sqrt{2}}\left(\frac{a}{\sqrt{3} t}\right) \frac{M_{\mathrm{s}}}{\mu_{\mathrm{B}} g \|}\left(c D_{\mathrm{nn}} S a\right)
$$

and in terms of the interfacial DMI constant $D_{\mathrm{DMI}}^{\mathrm{int}}$ we have $D_{\mathrm{DMI}}^{\mathrm{int}}=t \sqrt{3} / a D_{\mathrm{DMI}}$, which yields

$$
D_{\mathrm{DMI}}^{\mathrm{int}}=\frac{3}{2 \sqrt{2}} \frac{M_{\mathrm{s}}}{\mu_{\mathrm{B}} g \|}\left(c D_{\mathrm{nn}} S a\right)
$$

In the main part of the manuscript we calculate the exchange constant:

$$
A=\frac{M_{\mathrm{s}} a^{2} S J_{\mathrm{nn}}}{g^{\|} \mu_{\mathrm{B}}}
$$

The ratio of the interfacial DMI constant and the exchange constant can now be related to the fundamental quantities of the symmetric and antisymmetric exchange:

$$
\frac{D_{\mathrm{DM}}^{\mathrm{int}}}{A}=\frac{3}{2 \sqrt{2}} \frac{1}{a}\left(\frac{D_{\mathrm{nn}}}{J_{\mathrm{nn}}}\right)
$$

Thus, if the antisymmetric exchange simply scales in proportion to the symmetric exchange for a given material system, the ratio of the interfacial DMI and the exchange constant should be constant.

Non-reciprocal spin-wave modes. For thin magnetic films, the DE spin-wave modes are non-reciprocal - that is, the mode is localized either at the top or bottom interface-depending on its propagation direction and the orientation of $\mathbf{M}$. Hence, spin waves with $k>0$ are localized at the bottom (top) interface for $\mathbf{M} \|+z$ $(\mathbf{M} \|-z)$ and spin waves with $k<0$ are localized at the top (bottom) interface for $\mathbf{M} \|+z(\mathbf{M} \|-z)$. Thus, if the two $\mathrm{Ni}_{80} \mathrm{Fe}_{20}$ interfaces were to have strongly differing magnetic properties, it is possible that the non-reciprocal nature of the DE modes could also give rise to an asymmetric spin-wave dispersion. In the following, we will estimate an upper value for the frequency shift due to the non-reciprocal character of the surface waves.

For the geometry used here, the spin wave propagating in the positive $x$-direction is localized at the Pt interface for positive applied field, whereas the spin wave propagating in the opposite direction is localized at the SiN interface. If we neglect the symmetric-exchange contribution-that is, the long-wavelength limit-the DE-mode amplitude decays exponentially into the film thickness with a decay length $=1 / k$. For the wavevector $k=16.7 \mu \mathrm{m}^{-1}$ of the spin waves, which are measured in the BLS experiment, this results in a decay length $\delta=60 \mathrm{~nm}$. This yields a difference of the spin-wave amplitude between the two interfaces of 2 and $20 \%$ for the thinnest and thickest sample of the $\mathrm{Ni}_{80} \mathrm{Fe}_{20} / \mathrm{Pt}$ series, respectively. The Pt interface induces a thickness-dependent perpendicular anisotropy field $H_{k}$ (see Supplementary Fig. 4 in the Supplementary Information). Thus, the frequency of the spin wave with stronger localization at the Pt interface is shifted to lower frequencies with respect to the spin wave propagating in the opposite direction. To calculate the frequency shift due to the surface anisotropy we use a mean-field approach. The anisotropy field $H_{k}^{\text {int }}(t)$ is assumed to be localized within the first 
monolayer of the $\mathrm{Ni}_{80} \mathrm{Fe}_{20}$ layer. The effective anisotropy for each spin wave propagating in the positive and negative directions, respectively, is then calculated by weighting the anisotropy field with the spin-wave amplitude for the respective mode profile $m^{ \pm}(t)$ :

$$
H_{k}^{\text {eff }}=\frac{\int H_{k}^{\text {int }} m^{ \pm}(t) \mathrm{d} t}{\int m^{ \pm}(t) \mathrm{d} t}
$$

The integrals extend over the $\mathrm{Ni}_{80} \mathrm{Fe}_{20}$ layer thickness. The estimate of the frequency difference for the spin waves propagating in opposite directions is then calculated with equation (5). We find a frequency shift for the samples of about $0.03 \mathrm{GHz}$, almost independent of $\mathrm{Ni}_{80} \mathrm{Fe}_{20}$ thickness. Note that this is in qualitative contrast to the strong dependence of the DMI-induced frequency shift on $\mathrm{Ni}_{80} \mathrm{Fe}_{20}$ thickness (see Fig. 3 in the main text). The frequency shift due to the surface anisotropy would be even smaller if exchange were taken into account, because this would lead to a more gradual decrease of the spin-wave amplitude from the respective surface. Hence the value of $0.03 \mathrm{GHz}$ is an upper limit for the frequency shift due to the localization of the Damon-Eshbach modes. This upper limit is included in the error bars in Fig. 3 in the main text. The frequency shift due to the non-reciprocal character of the surface spin waves thus has only a very marginal influence on the frequency shifts determined by BLS in our measurements.

\section{References}

30. Demokritov, S. \& Tsymbal, E. Light scattering from spin waves in thin films and layered systems. J. Phys. Condens. Matter 6, 7145-7188 (1994).

31. Stamps, R. L. \& Hillebrands, B. Dipolar interactions and the magnetic behaviour of two-dimensional ferromagnetic systems. Phys. Rev. B 44, 12417-12423 (1991) 